\title{
MULTIVIEW REGISTRATION OF CARDIAC TAGGING MRI IMAGES
}

\author{
Estanislao Oubel ${ }^{a}$, Mathieu De Craene $^{a}$, Mattia Gazzola $^{a}$, Alfred O. Hero ${ }^{b}$ and Alejandro F. Frangi ${ }^{a}$ \\ ${ }^{a}$ Pompeu Fabra University, Department of Technology, Barcelona, Spain \\ ${ }^{b}$ The University of Michigan, Department of EECS, Ann Arbor (MI), USA
}

\begin{abstract}
This paper introduces a new method based on k-Nearest Neighbors Graphs (KNNG) for bringing into alignment multiple views of the same scene acquired at two different time points. This framework is applied to cardiac motion estimation from tagging MRI sequences. Features acquired in each view are collected in a high dimensional feature space and an efficient estimator of $\alpha$ - Joint Entropy ( $\alpha \mathrm{JE}$ ) is used for selecting the optimal alignment. In order to register 4D datasets, an analytical expression of the $\alpha \mathrm{JE}$ estimator was derived, enabling a fast implementation of gradient based optimization. The technique was tested in a set of six sequences and the results compared with respect to manual measurements made at tag crossing points, obtaining good accuracy and low processing times compared to published state of the art methods.
\end{abstract}

Index Terms - Image registration, Magnetic resonance cardiography, Motion analysis, Optimization methods, Entropy

\section{INTRODUCTION}

Tagged magnetic resonance imaging (MRI) is a well established technique used to obtain regional information on the left ventricle (LV) deformation [1], and thus potentially valuable for diagnosis of cardiovascular diseases. Basically, this technique consists in perturbing the magnetization of the myocardium in a specific spatial pattern at End-of-Diastole (ED). These perturbations appear as dark stripes (tags) when imaged immediately after application of the magnetic field. Since the myocardium retains "memory" of this disturbance, tags undergo the same deformation as the heart does, allowing to track material points inside the myocardium.

Mutual information (MI) based registration methods have been reported to be accurate for estimating cardiac motion fields in the left ventricle (LV) [2, 3]. The main drawbacks of previous approaches, like optical flow and tag detection and tracking, have been reported in [2]. Registration based method consist in applying non-rigid transformations to match the SA and LA views of a given cardiac phase, with their counterparts at ED. In [3] the authors propose to maximize a weighted average of the NMIs measured in the SA and LA views separately. This solution attempts to find a compromise between the alignment of each view. If the metrics have their optimum at different locations in the parameters space, the optimization could be driven by only one term of this linear combination or get trapped into a local maximum.

This paper introduces a similarity measure which incorporates measurements from both views simultaneously. $\alpha \mathrm{JE}[4]$ is computed from feature vectors concatenating the voxel intensities provided by SA and LA views. The proposed method estimates probability densities from $\mathrm{kNNG}$ [4] which allows to extend the methodology to an arbitrary number of views (e.g radial acquisitions). Even though this work is focused on cardiac motion estimation, the technique can be extended to a wide range of applications. Candidate applications are intersubject registration of images obtained with different imaging modalities (e.g. T1 and T2 sequences in brain scans), registration based on features like in $[5,6]$ and estimation of $3 \mathrm{D}+\mathrm{t}$ deformations using measurements performed on $2 \mathrm{D}$ views.

The next section introduces the general method and shows its application to cardiac motion estimation. Section 3 describes the data acquisition process and the experiments. Section 4 describes results for the tagging MRI application among with a short discussion. Finally, Section 5 draws conclusions and introduce future work.

\section{METHOD}

\subsection{Multiview registration framework}

Image registration methods aim to find the transformation $T$ : $\mathbf{X}_{f} \rightarrow \mathbf{X}_{m}$ maximizing a similarity criterion between a moving image $I_{m}\left(\mathbf{X}_{m}\right)$ and a fixed image $I_{f}\left(\mathbf{X}_{f}\right)$. Sometimes, the object under study is represented by more than one channel, each one providing different and complimentary information. In such situation the scenario consists in two sets of $\mathrm{N}$ fixed and moving images $\mathcal{F}=\left\{I_{f}^{1} \ldots I_{f}^{N}\right\}$ and $\mathcal{M}=$ $\left\{I_{m}^{1} \ldots I_{m}^{N}\right\}$ which can be thought of as images of vector elements $\mathbf{I}_{f}$ and $\mathbf{I}_{m}$. The main problem with the registration of such images is the metric computation. Similarity metrics derived from information theory have been successfully applied in medical imaging, but their computation for images of vectors is problematic, since they require to estimate probability density functions in high dimensional spaces. The estimators presented in [4] compute $\alpha \mathrm{JE}$ from measurements on graphs 


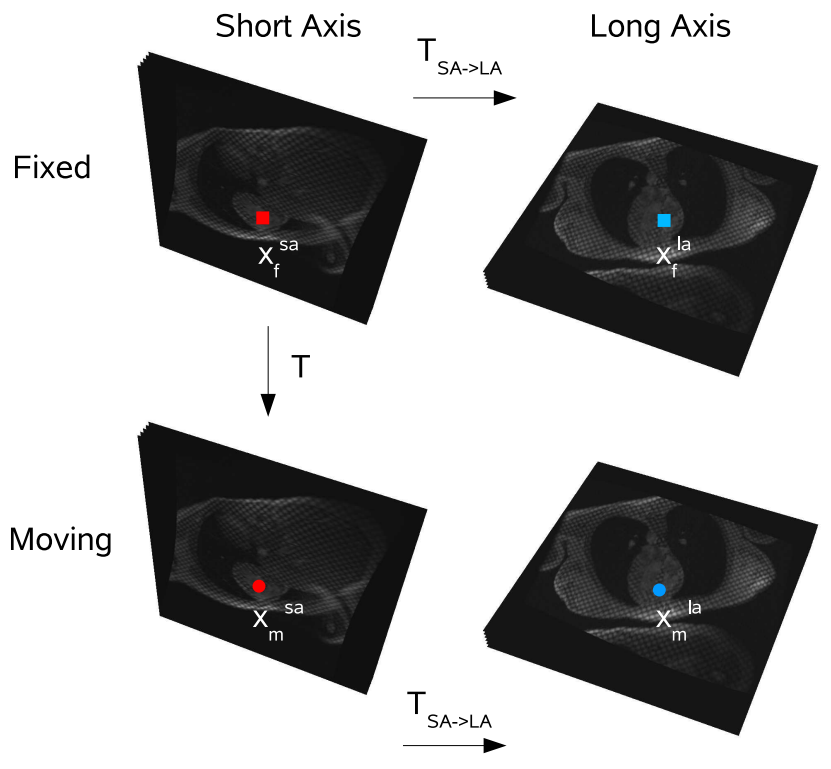

Fig. 1. Transformations involved in the method. The red square represents a point taken randomly in the fixed SA image and is placed in the other images by using the transformations. All positions correspond to the same material point.

directly computed in the feature space. These estimators are particularly suitable for estimating information theory quantities in high dimensional spaces and therefore have been selected in this multiview registration framework.

In the rest of the section, the general concept explained before is realized for cardiac motion estimation from tagging MRI sequences. In this specific case there are two fixed images and two moving images corresponding to SA and LA views of the heart. The image $\mathbf{I}_{f}=I_{f}^{s a}+\mathbf{i} I_{f}^{l a}$ is formed by $2 \mathrm{D}$ vectors resulting from the concatenation of voxel intensity in SA and LA views at ED. In the same way, the image $\mathbf{I}_{m}=I_{m}^{s a}+\mathbf{i} I_{m}^{l a}$ is formed by $2 \mathrm{D}$ vectors containing voxel intensities in SA and LA views at the phase for which the deformation is being estimated. Figure 1 shows all the transformations involved in this application.

\subsection{Cardiac motion estimation}

The motion estimation starts registering the second phase $\mathbf{I}_{1}$ of the cardiac sequence to the first one $\mathbf{I}_{0}$, which in general corresponds to ED. As a result, the first transformation $T_{0 \rightarrow 1}$ is obtained and allows to compute the displacement $\mathbf{I}_{0}$ to $\mathbf{I}_{1}$ from providing the displacements from ED to the next phase. Then, the third phase $\mathbf{I}_{2}$ is registered to $\mathbf{I}_{0}$ starting from the parameters defining $T_{0 \rightarrow 1}$, and a new transformation $T_{0 \rightarrow 2}$ is generated. This process is repeated for the rest of the phases $\mathbf{I}_{3} \ldots \mathbf{I}_{M}$ in the cardiac cycle. Once all the $\mathbf{M}$ phases are registered to the first one, the set of transformations
$\mathcal{T}=\left\{T_{0 \rightarrow 1} \ldots T_{0 \rightarrow M}\right\}$ model the myocardium deformation. The deformations undergone by the myocardium at each phase were modeled by means of Free-Form Deformations (FFD). This type of transformation was proposed by Rueckert et al. [7] for detection of cancerous lesions in contrast enhanced MR breast images, and has been succesfully applied in medical imaging applications.

\subsection{Combination of views}

During each iteration of the registration procedure, it is necessary to measure samples of vectors $\phi_{f}=\left[I_{f}^{s a}\left(\mathbf{x}_{f}^{s a}\right) I_{f}^{l a}\left(\mathbf{x}_{f}^{l a}\right)\right]$ and $\phi_{m}=\left[I_{m}^{s a}\left(\mathbf{x}_{m}^{s a}\right) I_{m}^{l a}\left(\mathbf{x}_{m}^{l a}\right)\right]$ concatenating voxel intensities provided by SA and LA for the fixed and moving images respectively. Thus, for each point $\mathbf{x}_{i}=\mathbf{x}_{i}^{s a}$ in SA in the image coordinate system, the corresponding one in LA must be found. The correspondence between points in SA and LA was obtained from DICOM format files which provides fields specifying origin and orientation of the images with respect to the coordinate system of the scanner. The points in world coordinates $\mathbf{x}_{w}^{s a}$ and $\mathbf{x}_{w}^{l a}$ corresponding to $\mathbf{x}_{i}^{s a}$ and $\mathbf{x}_{i}^{l a}$ in the local coordinate system of the image can be obtained in the following way:

$$
\begin{gathered}
\mathbf{x}_{w}^{s a}=\mathbf{o}_{w}^{s a}+\mathbf{D}_{w}^{s a} \mathbf{x}_{i}^{s a} \\
\mathbf{x}_{w}^{l a}=\mathbf{o}_{w}^{l a}+\mathbf{D}_{w}^{l a} \mathbf{x}_{i}^{l a}
\end{gathered}
$$

where $\mathbf{D}_{w}$ is the direction matrix having as columns the direction vectors $\mathbf{i}, \mathbf{j}$ and $\mathbf{k}$ expressed in the scanner coordinate system and $\mathbf{o}_{w}$ is the origin of the image in the same reference.

If $\mathbf{x}_{w}^{s a}$ and $\mathbf{x}_{w}^{s a}$ are the same point, the previous equations can be combined to obtain the transformation from SA to LA needed to generate the feature vectors.

$$
\mathbf{x}_{i}^{l a}=\left(\mathbf{D}_{w}^{l a}\right)^{T} \mathbf{D}_{w}^{s a} \mathbf{x}_{i}^{s a}+\left(\mathbf{D}_{w}^{l a}\right)^{T}\left(\mathbf{o}_{w}^{s a}-\mathbf{o}_{w}^{l a}\right)
$$

\subsection{Metric estimation}

Once the samples are generated, the similarity metric needs to be computed. In this work, the $\alpha \mathrm{JE}$ of feature vectors $\phi_{f m}=\left[\phi_{f} \phi_{m}\right]$ in $\mathbb{R}^{4}$ was employed and estimated by using the following estimator for a random variable $\mathrm{X}$ :

$$
\widehat{\alpha J E}(X)=\frac{1}{\alpha-1} \log E\left\{f(X)^{\alpha-1}\right\}
$$

In Eq. 4, the expectation operator is estimated from a sample of $n$ observations $\mathcal{X}=\left\{x^{1} \ldots x^{n}\right\}$ using the arithmetic mean over all observations. In our application, each sample $\phi_{f m}^{i}$ contains two pairs of SA and LA intensities in the fixed and the moving image domains. Using the same approach as in 
[4] for estimating the probability density from a kNN graph, the following estimator of $\alpha \mathrm{JE}$ in the joint feature space can be derived

$$
\widehat{\alpha J E}=\frac{1}{\alpha-1} \log \frac{1}{n^{\alpha}} \sum_{i=1}^{n}\left(\Gamma^{i p}\right)^{2 \gamma}
$$

where $\Gamma^{i}=\frac{1}{k} \sum_{p=1}^{k}\left\|\phi_{f m}^{i}-\widehat{\phi}_{f m}^{i p}\right\|$ and $\gamma=d(1-\alpha)$. In Eq. (5) $\widehat{\phi}_{f m}^{i p}$ represents the $p$-nearest neighbor of point $\phi_{f m}^{i}, d$ is the dimension of $\phi_{f}\left(\phi_{m}\right)$, and $\|\cdot\|$ is the euclidean distance.

\subsection{Analytical derivatives}

Many optimizers need to estimate the gradient of the cost function. The simplest approach is the use of finite differences, but this is computationally expensive for a high number of parameters. A more efficient way of calculating the gradient is to derive an analytical expression of the metric gradient, which is particularly important when using kNNG estimators since a kd-tree must be calculated before the nearest neighbor queries, which has time complexity $O(n \log n)$. The re-computation of the whole tree for each perturbation of the current set of parameters using finite differences would be unrealistically expensive, because of the high dimension of the FFD parameters space. Deriving the estimator in Equation 5 with respect to the parameter $p_{j}$ of the transformation, and assuming that the graph topology does not change (i.e. the neighbors of each point remain the same) for infinitesimal changes, the following gradient estimator can be obtained

$$
\frac{\partial}{\partial p_{j}} \widehat{\alpha J E}=\frac{2 d \sum_{i=1}^{n}\left(\Gamma^{i p}\right)^{2 \gamma-1} \frac{1}{k} \sum_{p=1}^{k} \frac{\partial}{\partial p_{j}}\left\|\phi_{f m}^{i}-\widehat{\phi}_{f m}^{i p}\right\|}{\sum_{i=1}^{n}\left(\Gamma^{i p}\right)^{2 \gamma}}
$$

where the partial derivative regarding the transformation parameter can be expanded as

$\frac{\partial}{\partial p_{j}}\|\cdot\|^{2}=\frac{\left(\left(I_{m}^{s a}-\widehat{I}_{m}^{s a}\right) \nabla I_{m}^{s a}+\left(I_{m}^{l a}-\widehat{I}_{m}^{l a}\right) \nabla I_{m}^{l a} J_{T_{s a \rightarrow l a}}\right) J_{T}}{\left\|\phi_{f m}^{i}-\widehat{\phi}_{f m}^{i p}\right\|}$

In the last expression, $J_{T}=\frac{\partial T(\mathbf{x})}{\partial p_{j}}$ and $J_{T_{s a 2 l a}}$ is the Jacobian of the transformation from SA to LA.

\section{EXPERIMENTS}

\subsection{Dataset}

Six tagged 4D sequences of healthy volunteers were acquired using a GE Genesis Signa 1.5T MRI scanner using a protocol specifically designed for the CDTEAM national project lead by the Pompeu Fabra University. A cine breath-hold sequence with a SPAMM grid tag pattern was applied, starting at ED and obtaining a total amount of 30 phases along the cardiac cycle. The image resolution for each phase was $0.78 \mathrm{~mm} \times 0.78 \mathrm{~mm} \times 8 \mathrm{~mm}$ and tags were placed every $5 \mathrm{~mm}$. In the deformation analysis only the systolic phase was used, since after that the tags vanish completely and therefore it is not possible to track material points inside the myocardium. The lenght of this part varies from patient to patient but is 10 phases approximately. In order to estimate deformations in the LV only, masks were defined manually at ED. One of the sequences was descarted because of the presence of severe acquisition artifacts.

\subsection{Evaluation}

In order to compute the error of the current method for tagging MRI, a set of points were defined at ED and End-ofSystole (ES). These points were marked manually at tags crossing points, since these are the only ones that can be put in correspondence in a confident manner. The fading effect of the magnetization field, the movement of the myocardium through acquisition planes, and local interactions in the tissue blur the image at ES and it is not possible to find the corresponding point for each point at ED. Thus, a selection of points was carried out to increase the accuracy in the error estimation. For the SA view, points were marked in the LV on planes localized just below the mitral valve, close to the apex and in a plane between these. For the LA view, only the central plane was used since the inter plane movement of the heart does not allow to define points correctly. This effect is atenuated in the central plane. An average amount of 18 points were marked for each patient.

\section{RESULTS AND DISCUSSION}

Figure 2 shows these errors for displacements estimated from SA landmarks and LA landmarks. This figure shows that the error in SA accumulated during the whole cardiac systole is lower than $1.6 \mathrm{~mm}$ for almost all cases, whereas this error increases for LA view. There are two (possibly combined) explanations to this observation: (a) tag crossings points were more difficult to define in LA than in SA because of differences in image quality. It was observed inter-slice movement of tissue as well (a material point is imaged in different slices along the cardiac cycle), which makes more difficult to track material points. (b) The method interpolates twice in LA (in fixed and moving images) whereas just once in SA (in moving image). For avoiding the introduction of unrealistic intensity values in the density estimation, one could consider to use the intensity of the closest point for the LA view or to use an interpolation method based on graphs analogous to partial volume techniques used for histograms.

Figure 3 displays graphically an example of the estimated deformation fields obtained. A visual analysis of these representations shows that all the basic components of the cardiac contraction are recovered (longitudinal/radial/circunferencial 


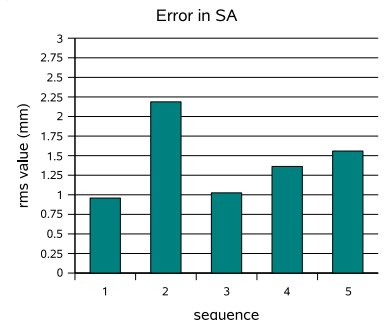

(a)

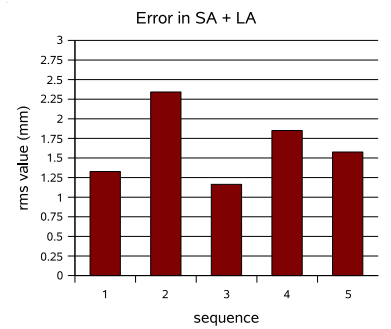

(c)

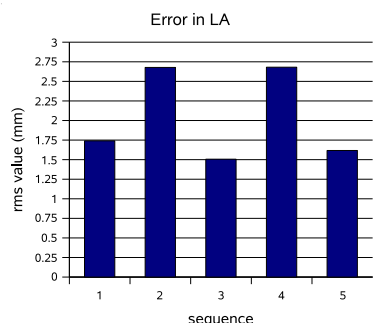

(b)

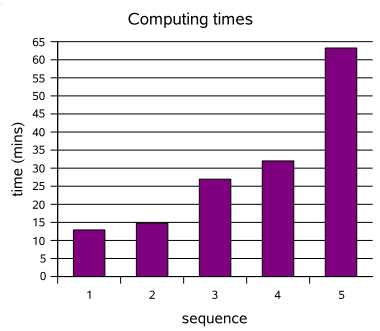

(d)
Fig. 2. Errors between displacements assesed manually and the estimated ones for (a) SA (b) LA and (c) SA + LA. (d) Computation for a 64-bits Itanium II processor at $1.5 \mathrm{MHz}$.

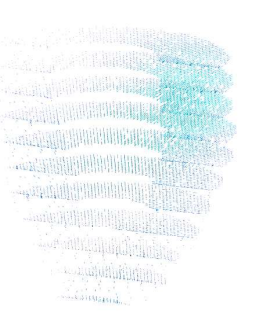

(a)

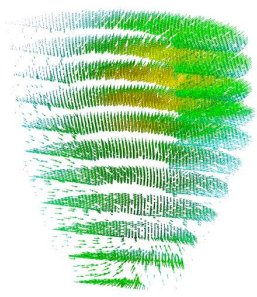

(b)

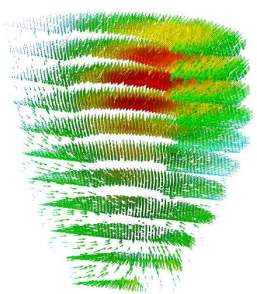

(c)
Fig. 3. Arrow plots of the estimated deformation fields at the (a) begining (b) middle and (c) end of the cardiac systole for the sequence 1 of the dataset.

shortening and torsion). This illustrates that the unified metric in 5 does effectively combine information from SA and LA views.

Differences in computation time (as plotted in Figure 2 (d)) are related to different image sizes, image resolutions and heart volumes in the population (since the number of points used for estimating the metric is proportional to the size of the region circumscribing the heart).

\section{CONCLUSIONS}

In this paper, a new method for multiview registration was presented. Its application to cardiac motion estimation from tagging MRI sequences provided accurate results over-performing the ones reported in [3]. An analytical formulation of the derivative for KNNG $\alpha$ JE estimator allows to speed up notably the optimization process (compared to finite differences) and lower the registration times to manageable values. The extension of this framework to other clinical problems is a topic of future research.

\section{REFERENCES}

[1] L. Axel and L. Dougherty, "MR imaging of motion with spatial modulation of magnetization," Radiology, vol. 171, no. 3, pp. 841-845, 1989.

[2] C. Petitjean, N. Rougon, F. Prêteux, Ph. Cluzel, and Ph. Grenier, "Measuring myocardial deformations from MR data using information-theoretic non rigid registration," in Proceedings Second International Workshop on Functional Imaging and Modeling of the Heart (FIMH'2003), Lyon, France - Lecture Notes in Computer Science 2674, Heidelberg, Germany, 5-6 June 2003, pp. 162-172, Springer Verlag.

[3] R. Chandrasheckara, R. H. Mohiaddin, and D. Rueckert, "Analysis of 3D myocardial motion in tagged MR images using non rigid image registration," IEEE Trans. Med. Imag., vol. 23, no. 10, pp. 1245-1250, October 2004.

[4] H. F. Neemuchwala and A. O. Hero, Multi-sensor image fusion and its applications, chapter Entropic graphs for registration, Marcel-Dekker, Inc., 2004.

[5] E. Oubel, C. Tobon-Gomez, A.O. Hero, and A.F. Frangi, "Myocardial motion estimation in tagged MR sequences by using aMI-based non rigid registration," in Medical Image Computing and Computer-Assisted Intervention, J. Duncan and G. Gerig, Eds., Palm Springs, CA, USA, October 2005, vol. 3750 of Lecture Notes in Computer Science, pp. 271-278, Springer.

[6] E. Oubel, A.O. Hero, and A.F. Frangi, "Complex wavelets for registration of tagged mri sequences," in IEEE International Symposium on Biomedical Imaging: From Nano to Macro, Arlington, VA, USA, April 2006, pp. 622-625, IEEE.

[7] D. Rueckert, L. I. Sonoda, C. Hayes, D. L. G. Hill, M. O. Leach, and D. J. Hawkes, "Nonrigid registration using free-form deformations: Application to breast MR images," IEEE Transactions on Medical Imaging, vol. 18, no. 8, pp. 712-721, Aug. 1999. 